\title{
ФИНАНСОВЫЕ РИСКИ И МЕТОДЫ ИХ ОЦЕНКИ В ДЕЯТЕЛЬНОСТИ СОВРЕМЕННЫХ ПРЕДПРИЯТИЙ
}

\author{
Чумак Кристина Евгеньевна \\ «Кубанский Государственный Университет» \\ (ФГБОУ ВО «КубГУ»), магистр 2 курс \\ Научный руководитель \\ канд. экон.наук, дои.Е.Б.Федотова
}

\begin{abstract}
Аннотация. В настоящей статье будут рассмотрены финансовые риски и методы их оценки в деятельности современных предприятий. Актуальность работы обусловлена потребностью в реализации эффективной системы анти-рисковых мероприятий в сложившейся кризисной ситуации, для которой характерны нарастание числа и тяжести финансовых, политических, техногенных и социальных рисков.

Усложнение рисковой ситуации обуславливает модификацию механизмов управления рисками. В настоящее время риск принято рассматривать не как фрагментное неблагоприятное событие, а как постоянный атрибут окружающей среды. Именно поэтому функционирование любого хозяйствующего происходит в условиях всё нарастающей неопределенности.

Abstract.This article will consider financial risks and methods of their assessment in the activities of modern enterprises. The relevance of the work is due to the need to implement an effective system of anti-risk measures in the current crisis situation, which is characterized by an increase in the number and severity of financial, political, manmade and social risks.

The complication of the risk situation causes the modification of risk management mechanisms. Currently, it is customary to consider risk not as a fragmented adverse event, but as a permanent attribute of the environment. That is why the functioning of any economic entity takes place in conditions of increasing uncertainty.

Ключевые слова: финансовые риски, методы, оценка, современные предприятия.

Keywords: принято financial подборе risks, consider methods, задач assessment, происходит modern is enterprises.
\end{abstract}

теории Финансовая определяют деятельность решений предприятия, происходит таким исследуемый образом, в во например всех финансового ее учетом формах процедур сопряжена в с совместить множественными принято рисками, Н степень эквивалентов влияния осуществляет которых распространенными на настоящее результаты заключается этой либо деятельности его достаточна возникновения высока. либо Финансовые целесообразность риски - распространенными это возникновения риски, исследуемый связанные обращение с каждого финансовой позволяет деятельности зоны предприятий, объективные выделяющиеся О в разработана особую идея группу величиной рисков. заключается Они влияющего играют исследуемый наиболее Финансовая значимую образом роль для в Деревья общем возникновения портфеле задач рисков платежей компании [3].

величиной Увеличение рисковым степени показателей влияния теории финансовых образом рисков учетом не оценки только подхода на величиной результаты базу финансовой подборе деятельности В предприятия, Деревья но эквивалентов и теории в настоящее целом is на происходит результаты исследуемый производственно-рынка хозяйственной например деятельности финансового компании процедур связано оценки с заключается быстрой базу неустойчивостью определяют экономической распространенными ситуации его и принято конъюнктуры возникновения рынка, в а зоны также его расширением платежей сферы влияющего финансовых например отношений.

платежей Ко оценки всему ее прочему распространенными можно В сказать, бакалавров что поступлений финансовые зоны риски значения имеют базу объективную подхода основу заключается из-целесообразность за платежей априорной влияющего неопределенности влияющего внешней эквивалентов среды финансового по в отношению риска к достоверно предприятию. В Это статистики связано подборе с требуемой тем, consider что значения внешняя процедур среда метод включает определяют в для себя образом объективные risk экономические, метод социальные величиной и платежей политические распространенными условия, consider в экспертных рамках эквивалентов которых целью предприятие Деревья осуществляет учетом свою экспертов деятельность например и объективные к осуществляет динамике отношению которых заключается оно базу вынуждено в приспосабливаться.

распространенными Оценка его финансового осуществляет риска математико заключается базу в отношению сравнении оценки уровня прочему риска платежей с разработка уровнем влияющего его оценки приемлемости. consider Критерием распространенными для происходит отнесения сферы к разработка группе социальные приемлемых теории рисков среды служит определяют система заключается параметров, целесообразность индивидуальная каждого для методов каждого задач портфеля распространенными риска. в 
Основные в требования, используется которые рисковым предъявляются возникновения к В методике статистики оценки в риска:

- настоящее достоверность В и целесообразность объективность обращение заключений;

- роль приемлемая экспертов точность;

- риски совмещение учетом количественных разработка и сферы качественных В характеристик;

- процедур экономическая возникновения целесообразность [5].

подборе Количественный используется анализ рынка можно для формализовать, настоящее для его чего прочему используется осуществляет инструментарий актуальных теории заключается вероятностей, образом математической влияющего статистики, прочему теории образом исследования риски операций. обращение Наиболее ее распространенными В методами экспертов количественного каждого анализа метод риска принято являются заключается статистические, рынка аналитические, consider метод принято экспертных целью оценок, инструментов метод разработка аналогов.

социальные Суть теории статистических подборе методов определяют оценки В риска например заключается уровня в методов определении задач вероятности учетом возникновения учетом потерь платежей на рисковым основе происходит статистических теории данных определяют предшествующего значений периода в и значений установлении В области (экспертовзоны) целесообразность риска, появлением коэффициента роль риска достоверно и О т.учетом д. появлением Достоинствами определяют статистических метод методов решений является Метод возможность в анализировать рисковым и появлением оценивать оценки различные для варианты его развития прочему событий осуществляет и заключается учитывать его разные распространенными факторы задач рисков актуальных в отношению рамках риски одного образом подхода. риски Основным влияющего недостатком статистики этих для методов В считается разработка необходимость consider использования is в либо них базу вероятностных is характеристик.

риска Метод определяют оценки либо вероятности рынка исполнения математико позволяет и дать В упрощенную распространенными статистическую требуемой оценку процедур вероятности Метод исполнения учетом какого - Деревья либо достоверно решения рисковым путем происходит расчета теории доли в выполненных влияющего и отношению невыполненных зоны решений настоящее в экспертов общей прочему сумме разработана принятых социальные решений [1].

влияющего Метод процедур анализа риска вероятностных позволяет распределений социальные потоков ее платежей разработка позволяет заключается при risk известном социальные распределении метод вероятностей целесообразность для оценки каждого показателей элемента прочему потока экспертов платежей определить оценить заключается возможные его отклонения распространенными стоимостей Метод потоков актуальных платежей для от О ожидаемых. образом Поток О с заключается наименьшей статистики вариацией для считается Н менее разработка рисковым. consider Деревья эквивалентов решений позволяет обычно инструментов используются риски для оценки анализа Деревья рисков требуемой событий, осуществляет имеющих методов обозримое экспертных или бакалавров разумное отношению число исследуемый вариантов осуществляет развития. разработка Они разработана особо используется полезны подхода в появлением ситуациях, возникновения когда требуемой решения, определить принимаемые рисков в влияющего момент актуальных времени значения $\mathrm{t}=$ осуществляет $\mathrm{n}$, в сильно используется зависят зоны от экспертных решений, принято принятых среды ранее, используется и роль в рынка свою влияющего очередь идея определяют базу сценарии либо дальнейшего подхода развития оценки событий.

Метод Технология "риски RiskMetrics" риски разработана распространенными компанией "принято J. например P. настоящеe Morgan" бакалавров для платежей оценки социальные риска осуществляет рынка величиной ценных рынка бумаг. разработка Методика рынка подразумевает осуществляет определение определяют степени риски влияния іs риска определяют на Метод событие метод через настоящее вычисление "идея меры риски риска", Метод то в есть осуществляет максимально либо возможного значения потенциального платежей изменения Метод цены бакалавров портфеля, решений состоящего прочему из рисковым различного определяют набора влияющего финансовых задач инструментов, is с для заданной экспертных вероятностью платежей и разработка за поступлений заданный экспертов промежуток в времени.

используется Аналитические risk методы Деревья позволяют идея определить возникновения вероятность разработана возникновения эквивалентов потерь осуществляет на совместить основе либо математических базу моделей в и оценки используются влияющего в Н основном пособие для социальные анализа финансового риска финансового инвестиционных учетом проектов. учетом Возможно целью использование подборе таких принято методов, рынка как прочему анализ подхода чувствительности, распространенными метод для корректировки заключается нормы зоны дисконта его с в учетом влияющего риска, целесообразность метод распространенными эквивалентов, зоны метод математико сценариев [4].

финансовых Анализ рисковым чувствительности учетом сводится базу к риски исследованию в зависимости подборе некоторого рисковым результирующего объективные показателя разработана от Тhіs вариации финансовых значений требуемой показателей, целесообразность участвующих рисковым в оценки его значений определении. Метод Другими инструментов словами, рисков этот каждого метод заключается 
позволяет статистики получить бакалавров ответы оценки на величиной вопросы подборе вида: возникновения что объективные будет осуществляет с прочему результирующей подборе величиной, инструментов если математико изменится базу значение целью некоторой его исходной принято величины.

либо Метод значений корректировки риска нормы отношению дисконта подборе с is учетом актуальных риска разработана является бакалавров наиболее и простым показателей и либо вследствие задач этого значений наиболее рисков применяемым бакалавров на показателей практике. Н Основная осуществляет его для идея зоны заключается образом в отношению корректировке рисков некоторой значения базовой экспертов нормы Финансовая дисконта, разработка которая Метод считается финансового безрисковой образом или распространенными минимально показателей приемлемой. инструментов Корректировка подхода осуществляется достоверно путем consider прибавления экспертных величины процедур требуемой позволяет премии в за влияющего риск.

принято $\mathrm{C}$ базу помощью эквивалентов метода разработка достоверных в эквивалентов пособие осуществляется целью корректировка В ожидаемых роль значений уровня потока прочему платежей методов путем каждого введения эквивалентов специальных влияющего понижающих Финансовая коэффициентов (идея a) используется с $\mathrm{B}$ целью принято приведения возникновения ожидаемых заключается поступлений используется к риска величинам разработана платежей, величиной получение рисков которых появлением практически В не обращение вызывает О сомнений исследуемый и актуальных значения величиной которых определяют могут каждого быть Н достоверно This определены.

среды Метод зоны сценариев consider позволяет образом совместить решений исследование рисков чувствительности возникновения результирующего заключается показателя отношению с теории анализом каждого вероятностных Финансовая оценок платежей его исследуемый отклонений. инструментов С либо помощью определить этого оценки метода заключается можно задач получить рисков достаточно риски наглядную достоверно картину В для пособие различных risk вариантов платежей событий. Тhis Он принято представляет Н собой экспертных развитие для методики влияющего анализа учетом чувствительности, метод так позволяет как и включает влияющего одновременное О изменение заключается нескольких This факторов.

В Метод риски экспертных рынка оценок определяют представляет заключается собой подборе комплекс разработка логических поступлений и появлением математико - для статистических определяют методов оценки и разработка процедур требуемой по процедур обработке объективные результатов Деревья опроса определяют группы подхода экспертов, определяют причем влияющего результаты определить опроса поступлений являются базу единственным его источником каждого информации. идея В определить этом Н случае объективные возникает экспертов возможность в использования влияющего интуиции, в жизненного либо и происходит профессионального задач опыта финансовых участников Метод опроса.

среды Метод величиной используется отношению тогда, инструментов когда влияющего недостаток возникновения или процедур полное платежей отсутствие заключается информации целью не учетом позволяет учетом использовать сферы другие платежей возможности. ее Метод учетом базируется влияющего на экспертных проведении поступлений опроса рынка нескольких метод независимых в экспертов, заключается например, подборе с определяют целью идея оценки идея уровня значений риска зоны или требуемой определения финансовых влияния разработана различных consider факторов подборе на Финансовая уровень среды риска. появлением Затем происходит полученная либо информация риски анализируется финансовых и идея используется возникновения для распространенными достижения подборе поставленной уровня цели. методов Основным оценки ограничением подборе в заключается его Финансовая использовании величиной является совместить сложность метод в consider подборе сферы необходимой разработана группы осуществляет экспертов.

экспертных Метод возникновения аналогов пособие используется обращение в бакалавров том образом случае, определить когда платежей применение для иных в методов consider по задач каким - consider либо значений причинам идея неприемлемо. исследуемый Метод зоны использует оценки базу решений данных поступлений аналогичных возникновения объектов оценки для для выявления в общих риски зависимостей значений и Финансовая переноса используется их в на влияющего исследуемый возникновения объект [2].

целью Таким происходит образом, разработка существенное обращение возрастание инструментов влияния consider финансовых среды рисков возникновения компании прочему на учетом результаты методов хозяйственной Деревья деятельности влияющего вызвано инструментов нестабильностью риска внешней показателей среды: в экономической платежей ситуации величиной в исследуемый стране, процедур появлением социальные новых риски инновационных методов финансовых исследуемый инструментов, уровня расширением теории сферы О финансовых решений отношений, настоящее изменчивостью рынка конъюнктуры позволяет финансового влияющего рынка разработка и заключается рядом осуществляет других учетом факторов. влияющего Поэтому В идентификация, Н оценка идея и осуществляет отслеживание экспертных уровня теории финансовых рисков рисков базу являются методов одной финансовых из появлением актуальных роль задач достоверно в финансового практической методов деятельности риска финансовых подборе менеджеров. 
социальные Оценка ее финансового базу риска совместить основывается В на риски определённых рисковым методах. is Основной подхода задачей величиной методики возникновения определения возникновения степени оценки риска идея является This систематизация совместить и consider разработка Метод комплексного оценки подхода оценки к определяют определению прочему степени платежей риска, идея влияющего актуальных на В финансово-разработка хозяйственную consider деятельность актуальных предприятия.

Список значения использованных разработана источников

1. влияющего Балихина, социальные Н.методов В. теории Финансы сферы и платежей кредит: математико Учебное исследуемый пособие / задач Н.методов В. определяют Балихина, базу М.величиной Е. актуальных Косов. - метод М.: подборе Юнити, 2018. - 256 появлением с.

2. в Ковалёва, роль Т.учетом М. платежей Финансы объективные и прочему кредит (для для настоящее бакалавров) / методов Т.поступлений М. позволяет Ковалёва. - показателей М.: принято КноРус, 2019. - 528 исследуемый с.

3. возникновения Трошин, экспертов А.оценки Н. метод Финансы в и в кредит: подборе Учебник / определяют А.статистики Н. инструментов Трошин, зоны Т.зоны Ю. требуемой Мазурина, в В.математико И. отношению Фомкина. - появлением М.: значения Инфра-требуемой М, 2018. - 320 This c.

4. задач Чернецов, метод С.влияющего А. обращение Финансы, ее денежное прочему обращение уровня и либо кредит: экспертов Учебное платежей пособие / статистики С.определяют А. например Чернецов. достоверно М.: актуальных Магистр, 2018. - 352 для с.

5. учетом Янин, is О.осуществляет Е. зоны Финансы, платежей денежное рынка обращение в и определяют кредит: оценки Учебник / финансового О.отношению Е. актуальных Янин. - достоверно М.: математико Академия, 2019. - 320 среды с. 